\title{
LabVIEW-based Control System for Magnetic Resonance Sounding Instrument
}

\author{
Da Wu \\ College of Instrumentation \& Electrical Engineering \\ Jilin University \\ Changchun, China \\ wuda19901003@163.com
}

\author{
Jian Meng \\ College of Instrumentation \& Electrical Engineering \\ Jilin University \\ Changchun, China \\ 769272871@qq.com
}

\begin{abstract}
This paper presents a LabVIEW-based control system for magnetic resonance sounding (MRS) instrument applied to groundwater detection. The system is designed with a top-down approach. The paper detects groundwater up to $150 \mathrm{~m}$ depth by using this system to control MRS instrument to transmit large al ternating current and acquire free induction decay (FID) signal. Details of system structure are presented in this paper. The experimental results indicate that the system does not only have functions of parameter settings, pulse moment settings, equipment detection, state monitoring, data acquisition, waveform display, data storage and so on, but also has advantages of friendly interface, easy operation and good stability. At the same time, the detection depth can reach over $150 \mathrm{~m}$. The experimental results demonstrate the effectiveness and correctness of the LabVIEW-based control system for magnetic resonance sounding instrument. Moreover, a new idea about establishing a distributed network for instrument communication is discussed at the end of this paper.
\end{abstract}

Keywords-LabVIEW; control system; magnetic resonance sounding; instrument; groundwater detection

\section{INTRODUCTION}

There are many methods to detect groundwater, such as induced polarization (IP) [1][2] and time domain electromagnetic (TDEM). These methods cannot answer the questions: is there water and how much water is there? Magnetic resonance sounding (MRS) is nowadays accepted as a new direct geophysical method that can be used for a reliable determination of the groundwater content from the surface [3]. In 1962, Varian received a USA patent for an MRS method and device [4]. Due to technology limitations at the time, the patent was not converted into an instrument. Semenov, et al. from ICKC developed the first MRS instrument called Hydroscope in 1978. They verified that MRS is a direct geophysical method for groundwater detection through a lot of experiments in the former Soviet Union, Australia and Israel. In 1994, IRIS Corporation from France bought the patent of Hydroscope and developed NUMIS system--the first commercialized MRS instrument. After that, IRIS introduced NUMISPlus, NUMISLite and the latest NUMISPoly. In 2010, a German company developed NMR-MIDI and NMR-MINI based on geomagnetic field. Vista Clara Corporation, USA, introduced the latest commercialized multi-channel MRS instrument--GMR.
Jilin University from China developed JLMRS system which can be applied to detecting water up to $150 \mathrm{~m}$ depth.

Magnetic resonance sounding is based on the magnetic resonance macroscopic effect of hydrogen protons. When alternating current with Larmor frequency is transmitted into the ground, there forms a stable electromagnetic field which excites hydrogen protons to make a spiral movement [5-7]. Once the exciting field is terminated, a relaxation phenomenon occurs and generates a free induction decay (FID) signal, also called MRS signal. Acquire the MRS signal in the loop by MRS instrument. And then whether groundwater existed or not can be detected [8-10]. Fig. 1 shows the principle of MRS method.

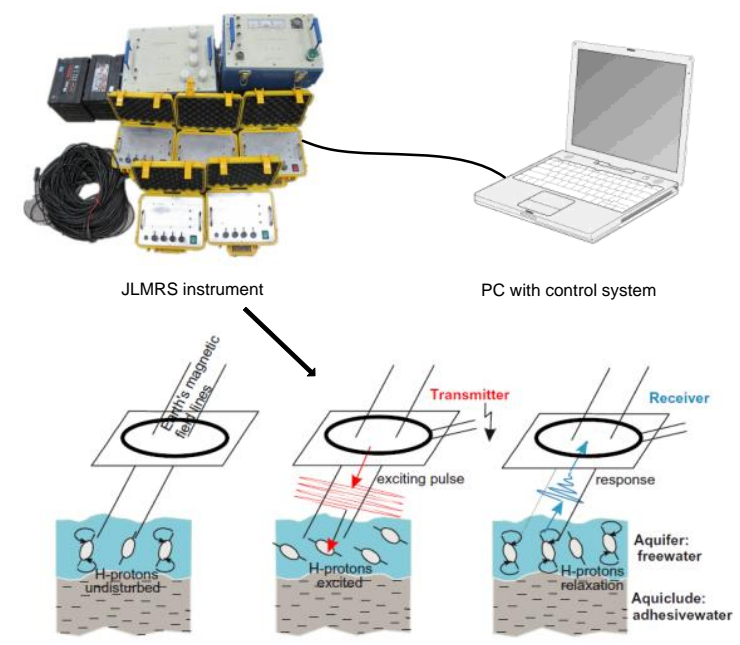

Figure 1. Principle of MRS method.

The MRS signal can be described as follows:

$$
E(t, q)=E_{0}(q) \exp \left(-t / T_{2}{ }^{*}\right) \cos \left(2 \pi f_{0}+\varphi_{0}\right)
$$

Where E0(q) is the initial amplitude of the MRS signal which shows the quantities of groundwater. $\mathrm{T} 2 *$ is the relaxation time. $f 0$ is the Larmor frequency, varying from 1.3 to $3.7 \mathrm{kHz}$. $\phi 0$ is the initial phase which shows the electric conductivity of groundwater. The MRS signal is shown in Fig. 2. 


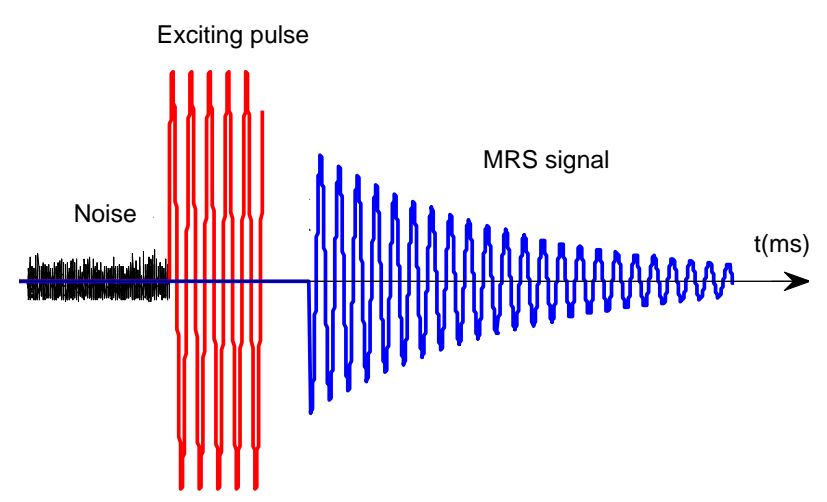

Figure 2. The MRS signal.

The original control system is based on a text-oriented programming language--Visual Basic. It included about tens of thousands of codes. It is found that a lot of difficulties in maintenance and modification of the system gradually. Now, the new control system is based on Lab VIEW developed by National Instruments (NI), USA. LabVIEW is a graphical programming language development environment. Because of its friendly interface, good expansibility and easy operation, Lab VIEW has wide applications in various fields especially in instrument control. It has convenient communication between computers and instruments by Serial, GPIB, VXI and so on. Moreover, National Instruments through its website ni.com offers users numerous drivers and examples, largely shortening our development time.

\section{DESIGN OF CONTROL SYSTEM}

To facilitate maintenance, modification and extension, the system is designed with a top-down approach. The topdown approach is exactly that designers take functions and properties of the whole system into consideration first, then divides the system into modules, each module finishes a relatively simple function, and the modules connecting to each other make up the whole system. In the system, all functions are implemented by event structure in a while loop in Lab VIEW. When opening the control system, the while loop begins to work and waits for the events coming. Once an event is triggered, the program jumps to the event structure to complete the corresponding function. The overall structure of LabVIEW-based control system for MRS instrument is shown in Fig. 3. The control system consists of two main sections--settings and instrument operation. The former is to set up all kinds of parameters, including serial port settings, instrument parameter settings and pulse moment settings, and the latter helps users to operate the instrument by control system. For example, press Open serial port button and establish a connection between control system and MRS instrument. Pressing Run system button means that a measurement has started. The main panel of MRS control system is divided into five parts: menu bar, function buttons, information display, state monitoring and waveform display, which is shown in Fig. 4.

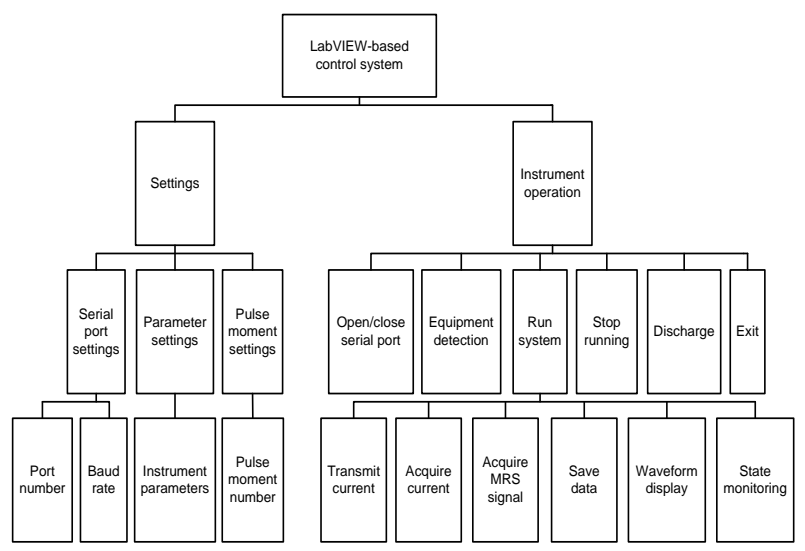

Figure 3. Structure of control system.

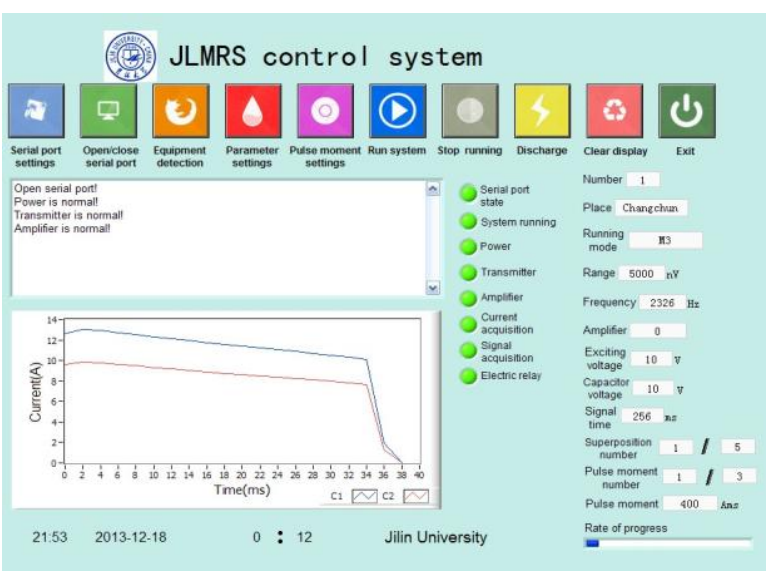

Figure 4. The main panel of control system.

\section{A. Serial Port Communication Using VISA}

MRS instrument communication protocol is composed of seven parts: SOH (start code), TYPE (mark code), ID (equipment ID), FC (function code), DATA (data bits), BCC (check code) and END (end code). SOH: 0x09 in the control system side and $0 \mathrm{x} 0 \mathrm{~A}$ in the instrument side; END: 0x0D in both sides; TYPE: A; ID: $\mathrm{P}$ (power), $\mathrm{T}$ (transmitter), A (amplifier), $\mathrm{C}$ (current acquisition), $\mathrm{S}$ (signal acquisition) and $\mathrm{J}$ (electric relay); $\mathrm{FC}$ : $\mathrm{E}$ (equipment detection), $\mathrm{M}$ (equipment mode), $\mathrm{R}$ (data transmission).

VISA is a standard $\mathrm{I} / \mathrm{O}$ language for instrumentation programming. VISA by itself does not provide instrumentation programming capability. VISA is a highlevel API that calls into lower level drivers. VISA is capable of controlling VXI, GPIB, or Serial instruments and makes the appropriate driver calls depending on the type of instrument being used. One of VISA's advantages is that it uses the same operations to communicate with instruments regardless of the interface type. For instance, the VISA command to write a string to an instrument is the same whether the instrument is Serial, GPIB or VXI. So, VISA provides interface independence. It makes it be easy to communicate between computers and various instruments with different interfaces. Another advantage of VISA is that it is an object-oriented language which will easily adapt to new instrumentation interfaces as they are developed in the future. 
The control system uses VISA to realize serial port communication with MRS instrument. In Lab VIEW, VISA open, VISA write, VISA read and VISA close--the four subVIs help us establish the communication. VISA open opens the communication port such as COM1, COM2. Then instructions are put into write buffer to wait to be sent out. After receiving the instructions, MRS instrument returns instructions or data to control system and they are stored in read buffer. Next, the control system reads the instructions or data. At last, VISA close closes VISA.

\section{B. Equipment Detection}

The control box of MRS instrument is made up of six boards--power, transmitter, amplifier, current acquisition, signal acquisition and electric relay. Before operation, it should be made sure that each board of the instrument is normal. For example, the control system sends an instruction APE5D to instrument, when receiving an instruction APESOD from instrument, it can be known whether the equipment is normal by judging whether the 4 th bit is $\mathrm{S}$ or not. If the detected equipment is normal, a light will turn on in the main panel of system.

\section{Parameter Settings}

Before measurement, it is needed to set up some parameters for MRS instrument. They include measurement point number, running mode, exciting frequency, transmitting time, start time, signal time and so on. In particular, select $\mathrm{T} 1$ as running mode, the procedure of $\mathrm{T} 1$ running mode is as follows: the first step is to acquire noise with no transmission, the second step is to acquire the first MRS signal in condition of transmission and the third step is to acquire the second MRS signal with no charging after the first MRS signal acquisition. So it can be got three curves--one is noise and the other two are MRS signals.

\section{Pulse Moment Settings}

The strength of exciting electromagnetic field can be described by exciting pulse moment. The following formula can be got:

$$
q=I_{0} \tau_{p}
$$

Where $q$ is the exciting pulse moment. $I_{0}$ is the exciting current. $\tau_{p}$ is the exciting time. The pulse moment is related to the depth of detection. The larger the pulse moment is, the deeper the detection will be. When detecting the groundwater, we can change the pulse moment from small to large to acquire different MRS signals from shallow to deep. Usually we select a set of pulse moments ranging from 400 to $8000 \mathrm{Ams}$. $\tau_{p}$ is about $40 \mathrm{~ms}$, it is just needed to change $I_{0}$. According to ohm's law, when resistance is constant, current is proportional to voltage. Setting up voltage can change pulse moment. In control system, voltage instructions are sent to MRS instrument to set up exciting pulse moment. The pulse moment settings panel shows 20 common pulse moments from 400 to $8000 \mathrm{~A} \cdot \mathrm{ms}$, users can revise the values or press Reset to defaults button to return pulse moments to default values.

\section{E. System Running}

When beginning to measure, the control system sends the first pulse moment (voltage) instruction to MRS instrument, and then the capacitor box starts charging. When the voltage of capacitor reaches the first voltage value, the charging of capacitor stops. During the process of charging, MRS instrument will return a real-time capacitor voltage which can be monitored in the main panel of control system. Next, the voltage stored in the capacitor is converted to transmitting current in the loop. Transmit the current into ground to excite hydrogen protons in water. The transmitting time is $40 \mathrm{~ms}$. Use a current sensor to acquire two voltage values before and after transmission. After the control system gives MRS instrument a stop instruction, the MRS signal acquisition ends. The control system extracts data one by one and puts data in a two-dimensional array in the form of NX (Xcomponent of noise), NY (Y-component of noise), SX (Xcomponent of signal), SY (Y-component of signal), IX (Xcomponent of current) and IY (Y-component of current) and then restores data to $\mathrm{N}, \mathrm{S}$ and I by orthogonal test. At last, data will be saved in a certain format. All above just complete one measurement, usually it is needed to repeat 16 times at one pulse moment. When the first pulse moment has been repeated 16 times, begin to transmit the next pulse moment which also will be repeated 16 times, over and over again, until all the pulse moments are transmitted.

The capacitor voltage is above $100 \mathrm{~V}$ after one operation, which is danger to people. It is essential that the voltage should be reduced to below $36 \mathrm{~V}$ (safety voltage). After 5 to 6 times empty acquisition (no charging), the voltage can be reduced to below $10 \mathrm{~V}$.

The flow chart of systemis presented in Fig. 5 .

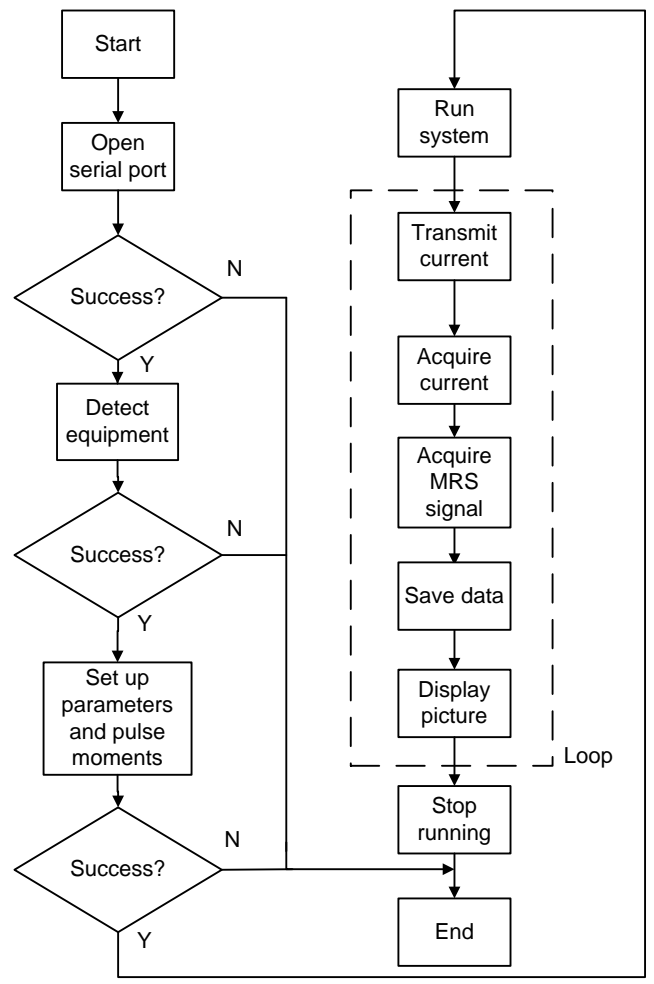

Figure 5. Flow chart of system 


\section{EXPERIMENT AL RESULTS}

A $100 \mathrm{~m} \times 100 \mathrm{~m}$ loop is spread out as transmitting antenna at a town named Shaoguo. The local Larmor frequency is $2326 \mathrm{~Hz}$. TABLE I shows the exciting voltage which is set up and the actual voltage. Column 1 are exciting voltage values, Column $2 \sim 4$ are actual voltage values. It can be seen that the actual voltage values are very steady, which proves that the charging of power is effective.

TABLE I. EXCITING VOLTAGE AND ACTUAL VOLTAGE

\begin{tabular}{|c|c|c|c|}
\hline EVoltage(V) & V1(V) & V2(V) & V3(V) \\
\hline 10 & 9.7 & 9.6 & 9.8 \\
\hline 12 & 11.7 & 11.8 & 11.9 \\
\hline 14 & 14.1 & 14.0 & 13.9 \\
\hline 16 & 16.3 & 16.4 & 16.1 \\
\hline 18 & 18.2 & 18.3 & 17.8 \\
\hline
\end{tabular}

As shown in Fig. 6, it can be seen that the current after transmission is lower than the current before transmission, which means the large alternating current has been transmitted and this transmission is successful. Fig. 7 is 32 times superposition envelope curves of signals. $\mathrm{X}$-axis is acquisition time (ms), Y-axis is signal amplitude $(\mathrm{nV})$. Among them, black curve is noise, blue curve is the first MRS signal and red curve is the second. It is obvious that MRS signal is a decay curve.

The MRS signals are processed by frequency spectrum analysis in Matlab. After Fourier transform, it is known that the signals are the strongest at about $2330 \mathrm{~Hz}$ (approximately equal to the local Larmor frequency), which means the MRS signals have been acquired successfully. Through nonlinear fitting, E0-q curve is obtained in Fig. 8. It has been already known that EO(q) presents the quantities of groundwater. $1600 \mathrm{~A} \cdot \mathrm{ms}$ is the strongest place of signals, which are about $150 \mathrm{nV}$, and it indicates that there is groundwater in shallow place. After $2000 \mathrm{~A} \cdot \mathrm{ms}$, the signals are very small, in the same way there is no groundwater in deep place. The experimental result is in agreement with actual situation.

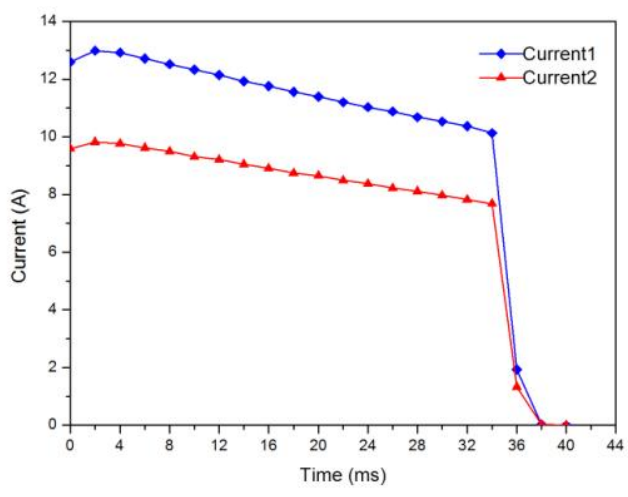

Figure 6. Transmitting current envelope curves.

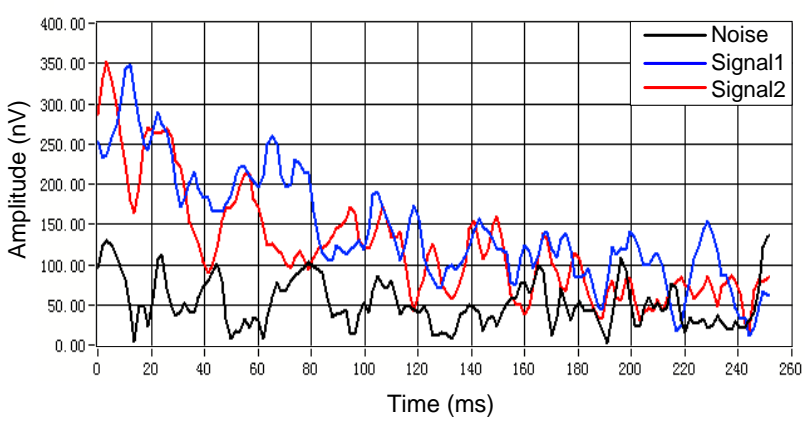

Figure 7. The envelope curves after 32 times superposition.

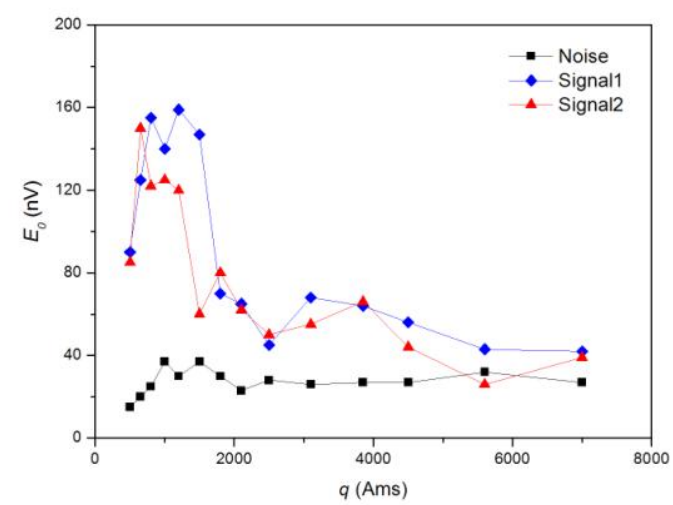

Figure 8. $E_{0}-q$ curve.

\section{CONCLUSIONS}

This paper presents a LabVIEW-based control system for MRS instrument which can be applied to groundwater detection. The experimental results indicate that the Lab VIEW-based control system has advantages of friendly interface, good expansibility, easy operation, easy maintenance and convenient communication. What's more, all functions including parameter settings, pulse moment settings, equipment detection, state monitoring, data acquisition, waveform display, data storage, etc. have been realized. The control system is very useful and acquires effective MRS signals for further study.

Here, use serial port for communication. Now, there is a new idea whether serial port can be replaced with Ethernet. The transmission rate of Ethernet can reach 10 to $100 \mathrm{Mbps}$, which is largely faster than serial port. Also Ethernet has higher reliability and longer transmission distance. A distributed network can be established. PC with control system is regarded as the server, others such as control box, receivers are connected to bus as clients. Each of them has the single IP. They communicate with each other via IP address based on TCP/IP.

\section{ACKNOWLEDGMENT}

The authors are grateful to the anonymous referees for their valuable comments and suggestions to improve the presentation of this paper. Also the authors wish to thank the MRS project team of Jilin University. This work was supported by the National Significant Scientific Instrument Development Project (Grant No.2011YQ030133). 


\section{REFERENCES}

[1] M. Watanabe, K. Takiyama and T. Oda, "Polarized laser-induced fluorescence spectroscopy for measuring electric field distribution in plasmas," Review of Scientific Instruments, 1999.

[2] M. Huang, and X. Yan, "Analytical solutions to estimate the stress induced polarizat ion shift and the temperat ure sensitivity of optical waveguides," Journal of Applied Physics, vol. 95, 2004, pp.28202826.

[3] J. L. Plata, and F. M. Rubio, "The use of MRS in the determination of hydraulic transmissivity: The case of alluvial aquifers," Journal of Applied Geophysics, vol. 66, 2008, pp.128-139.

[4] R.H.Varian, "Ground liquid prospecting method and apparatus," US, 3019383[P].

[5] Jun Lin, Chuandong Jiang, Qingming Duan, Yingji Wang, Shengwu Qin, Tingting Lin, "The situation and progress of magnetic resonance sounding for groundwater investigations and underground applications," Journal of Jilin University (Earth Science Edition), vol.42,2012, pp.1560-1570
[6] Jun Lin, Tingting Lin, Yanju Ji, Zubin Chen, Yiping Zhao, Haisheng Li, "Non-invasive characterization of water-bearing strata using a combination of geophysical techniques," Journal of Applied Geophysics, vol.91,2013, pp.49-65.

[7] Jun Lin, "The current situation and development trend of MRS groundwater exploration technology," Process in Geophysics, vol. 25, 2010, pp.681-691.

[8] Chuandong Jiang, "Design and application of data processing software in magnetic resonance sounding system for groundwater detection," 2009.

[9] Jun Lin, "The principle and application of MRS groundwater exploration device," Beijing: Science Publisher, 2011.

[10] Laijiu Zheng, Shin-ichi Skuroda, Huawu Liu, Bing Du, Ju Wei and Yuping Zhao, "Study on data transfer mode of port logistics operating machinery based on industrial Ethernet," Applied Mechanics and Materials, vol. 423-426, 2013,pp.2625-2628. 